\title{
Verbal Short-Term Memory as Language Predictor in Children with Autism Spectrum Disorder
}

\author{
Ioanna Talli \\ Department of Italian Language and Literature, Aristotle University of Thessaloniki, Thessaloniki, Greece \\ Email: tallijoan@yahoo.com
}

How to cite this paper: Talli, I. (2020) Verbal Short-Term Memory as Language Predictor in Children with Autism Spectrum Disorder. Journal of Behavioral and Brain Science, 10, 200-219. https://doi.org/10.4236/jbbs.2020.105013

Received: April 2, 2020

Accepted: May 15, 2020

Published: May 18, 2020

Copyright ( 2020 by author(s) and Scientific Research Publishing Inc. This work is licensed under the Creative Commons Attribution International License (CC BY 4.0).

http://creativecommons.org/licenses/by/4.0/

\begin{abstract}
Verbal short-term memory (vSTM) has been shown to be associated with language development in typical and atypical populations. In this study, we investigated cognitive and language skills in 33 school-aged children with ASD (6 - 12 years old) with both typical and low levels of intelligence (18 with typical non-verbal IQ [ $>80$ in Raven] and 15 with low non-verbal IQ [ $<60$ in Raven]). We administered standardized measures for the assessment of cognitive skills: non-verbal IQ (Greek version of Raven), vSTM (assessed with word recall), immediate and delayed visual memory, as well as language skills: expressive vocabulary (Greek Crichton vocabulary scale), receptive vocabulary and syntactic production (assessed with sentence recall). We were interested in investigating whether there is an interrelation between cognitive and language measures, which cognitive function(s) could better predict the language skills of children with ASD and vice versa and whether ASD children with low cognitive skills always perform poor on language tasks. The results showed that non-verbal IQ correlated significantly and strongly with all three language tasks $(p<0.001)$, while memory tasks (vSTM, immediate and delayed visual memory, visual information recall) correlated significantly and strongly with expressive vocabulary and syntactic production $(p<0.05)$. Regression analysis showed that expressive vocabulary was predicted by non-verbal IQ and vSTM, syntactic production was predicted by vSTM and picture comprehension was predicted by non-verbal IQ. Conversely, expressive vocabulary could predict non-verbal IQ, vSTM, immediate visual memory, delayed visual memory, and visual information recall. It seems that vSTM is a strong predictor of language skills for children with ASD, just like it is for other typical and atypical populations. Finally, dissociations exist in individual performances between non-verbal IQ and memory on the one hand and language skills (expressive vocabulary, syntactic production) on the
\end{abstract}


other hand. We discuss the significance of these findings in terms of previous results reported in ASD literature as well as in terms of clinical implications and intervention in ASD individuals.

\section{Keywords}

Autism Spectrum Disorder, Language Skills, Cognitive Skills, Verbal Short-Term Memory

\section{Introduction}

Autism Spectrum Disorder (ASD) is characterised by persistent deficits in social communication and social interaction and restricted and repetitive patterns of behavior in early developmental period, according to Diagnostic and Statistical Manual of Mental Disorders [1]. Along with these phenotypical autistic symptoms, variations in performance in cognitive skills-such as intelligence, memory and attention, as well as in language skills may also be present in ASD. Almost half (44\%) of children identified with ASD have average to above average intellectual ability (i.e., intelligence quotient [IQ] $>85$ ), $31 \%$ are classified in the range of intellectual disability (IQ $<70$ ) and $25 \%$ are in the borderline range (IQ 71 - 85) [2] [3].

The phenotype concerning how affected are cognitive and language skills in ASD varies dramatically in degrees of severity and impairment. However, very little is known about the interaction between these two domains. Children with ASD may display spared skills in some areas of cognitive functioning (such as in non-verbal or visuo-spatial domain) [4] [5] [6] and low level skills in others -such as in verbal abilities, working memory and processing speed [7] [8] [9].

\subsection{Language Skills in ASD}

There is great heterogeneity and variability in the language development in ASD and a large cohort of studies has shown that it is qualitatively similar in its developmental course to that of non-autistic children at the same stages of development (for a review see [10]). Language skills in ASD, unless they are intact, may be impaired across all domains with different patterns of strengths and weaknesses; however research has focused mainly in the domain of pragmatics and discourse, which are socially motivated language domains [11]. This is because even in cases where children no longer meet the criteria for ASD diagnosis (the so called "optimal outcome" children), pragmatics and discourse are still impaired [12] [13] [14]. Another reason why research hasn't focused on other domains of language, such as phonology or morpho-syntax is because, although they are important domains of language, deficits in such domains are not present in all individuals with ASD and are not considered necessary for an ASD diagnosis [15]. For instance, grammatical abilities in ASD vary widely, even in high-functioning (HF) individuals with ASD. Some children have average 
grammatical abilities [15] [16], while others have impaired grammatical skills [15] [17] [18] [19]. Moreover, a proportion of children with ASD fail to acquire spoken language skills and may use from zero to less than 20 - 30 words [20]. On the other hand, some studies investigated the resemblance of grammatical (morpho-syntactic) impairment in HF children with ASD and in children with DLD. Some found similar skills [21], others found more severe deficit in children with DLD [22], while others in children with ASD [23]. However the question of how language skills develop in low functioning ASD children remains open. There are studies that have found very impaired morpho-syntactic skills [24], while others have found spared language skills [25]. In Greek, Terzi and colleagues found impaired performance both in comprehension and production of clitic pronouns in ASD children with HF (aged 5 - 8 years) compared to TD vocabulary-matched children. The same results were found both when comprehension was assessed via a picture matching task [26] and production via a narration task (Frog, where are you?) [27].

It is thus, very important to investigate why language outcomes are so heterogeneous, in terms of individual performance and the degree to which language skills are impaired. Moreover, it is important to clear whether low cognitive skills in children with ASD indicate low language skills and conversely. As far as we know, very few studies assessing language skills in ASD have included both ASD children with normal and low cognitive abilities (such as non-verbal IQ and memory).

\subsection{Cognitive Skills in ASD}

Significant variability exists also across cognitive skills in ASD. First of all, intellectual ability (non-verbal IQ) may vary in individuals with ASD from average or above average level to intellectual disability. Of course, the characteristics of individuals with intellectual disability vary dramatically from those with average or above average non-verbal IQ skills in terms of linguistic, cognitive and social skills [28]. Previous research, especially genetic studies, has suggested that non-verbal IQ may constitute a basis for ASD subtypes [25] [29]. Nevertheless, for more detailed analysis of ASD profiles, when assessing non-verbal IQ, it is considered to be of crucial importance to assess verbal IQ as well [30].

Memory function in ASD has been a subject of study for decades and is often a combination of typical and atypical functioning. Early studies of memory skills in ASD have shown intact memory span (e.g. [31] [32] [33], however more recent studies have shown that children and adults with ASD have difficulties with STM, especially with serial order recall [8]. This variation in memory function patterns cannot be explained by intelligence or language skills, but is probably related to these skills [8] [34].

In addition, recent research has shown that individuals with ASD demonstrate difficulty in verbal and non-verbal STM, especially in serial order but not in item recall [8] [35] [36]. Several other studies have shown that ASD individuals have lower spatial STM and working memory (WM) span than TD groups (for a re- 
view see [37]).

Visual memory has been found to be an area of strength for children with ASD, but it depends on the complexity of the stimuli, whether they depend on verbal skills or not (e.g. meaningless shapes) and on the effect of age and IQ [38]. For instance, Ameli et al. [39] found poor visual memory skills for meaningless patterns, but normal for meaningful pictures. Other studies have linked visual memory skills (for meaningless patterns) with intelligence, since the differences between ASD and TD children disappeared when controlling for non-verbal IQ [40]. Contrary to this finding, Chien et al. [38] assessed two groups of children and adolescents with ASD, one with autism and one with Asperger's Syndrome (normal intelligence), based on the criteria of the previous version of DSM-IV, and a TD age-matched group on visual memory tasks and found that both clinical groups were worse than TD and that there was no significant difference in the performance between the two clinical groups, regardless of their difference in non-verbal IQ. They suggested that visual memory is impaired across all individuals with ASD, regardless of their IQ level. Funabiki and Shiwa [41] also found visual but not auditory WM deficits in individuals with ASD compared to the same-aged and the same full-scale IQ TD individuals. Consequently, there is no consistency in findings regarding visual memory, nor is it clear whether subgrouping reveals different cognitive abilities across participants with ASD.

\subsection{The Relation between Memory and Language Abilities in ASD}

As far as memory compared to language skills in ASD population are concerned, studies have come up so far with heterogeneous and contrasting results. STM and working memory have been suggested to be fundamental for language acquisition, both language reception and expression [42] [43] [44] [45]. More specifically, vSTM has been repeatedly found to be related to vocabulary development in typical and atypical populations (e.g. [46] [47] [48] [49] [50]).

Concerning the performance and the association between verbal STM (vSTM) and syntactic abilities in ASD, findings are conflicting. Some studies show that children with ASD have similar vSTM and syntactic skills with TD children. Faja and Dawson [51] compared vSTM abilities of 6 - 7 year old children with ASD to those of TD children and found no differences between the two groups. Furthermore, Phelan, Filliter, and Johnson [52], found no differences in performance on a verbal memory test (word recall; California Verbal Learning Test) between a group of ASD children and adolescents and TD children matched on age and non-verbal IQ.

Nevertheless, there are findings that show lower performance of ASD compared to TD children in vSTM and syntax. Gabig [53] found that children with ASD underperformed their age-matched TD children in both vSTM (repetition of non-words, digits) and syntactic production (sentence repetition), especially in sentence repetition, which is considered as a complex syntactic task, suggesting that language processing demands affect performance of ASD children on 
vSTM tasks. Moreover, Meir and Novogrodsky [9] assessed vSTM (forward digit span), WM and syntactic production skills (sentence repetition) of monolingual and bilingual children aged 4.6 - 9.2 years with HF autism and TD peers and found lower performance in HF children with ASD. However, some of monoand bilingual HF children with ASD had unimpaired syntactic abilities but no differences were found in vSTM skills between those with impaired and unimpaired syntactic skills. They suggested that syntactic difficulties of HF children with ASD do not stem from impaired vSTM skills. Likewise, Durrleman, Delage, Tuller and Prevost [54] did not find a relation between syntactic skills (assessed with a comprehension of passives task) and vSTM and WM skills.

Contrary to these findings, Tyson et al. [55] assessed two groups of ASD individuals, one with HF and one with optimal outcome autism (OO) and compared them with a TD group on language (syntax and semantics assessed with CELF-IV test), receptive vocabulary, vSTM (word recall) and phonological STM (non-word repetition) tasks. They found that HF group with ASD performed more poorly on all tasks than the $\mathrm{OO}$ and TD group and that for both clinical groups with ASD, vSTM and phonological STM skills predicted the language skills of both clinical groups -and a bit more of the OO group, but not of the TD group, suggesting that the good language abilities of participants with ASD with normal non-verbal IQ are a reflection of their good vSTM abilities. Similarly, Durrleman and Delage [17] showed that ASD children with impaired syntactic skills scored lower on a vSTM task than ASD children with unimpaired syntactic skills, demonstrating a link between syntactic abilities and vSTM.

Furthermore, given that VSTM is a clinical marker of language disorders, it has been suggested that a comorbidity exists with DLD in children with ASD who have language problems [56]. However, studies comparing both clinical populations have repeatedly shown that they are two distinct developmental disorders with distinct profiles of difficulties in VSTM [57] [58] [59].

Therefore, it is not yet clear: 1) whether good language abilities reflect good cognitive abilities, 2) whether language difficulties always co-exist with low cognitive abilities and 3) whether there are differences between these skills among subgroups of children with ASD. Our study aims besides the above questions at exploring whether cognitive abilities among children with ASD can predict language abilities and vice versa.

\subsection{The Present Study}

This study aims at contributing to the field of language skills in children with autism by examining the cognitive and language skills of school-aged children with ASD (6 - 12 years old) with typical and low levels of intelligence. More specifically, we assessed with standardized measures the following cognitive skills: non-verbal IQ, verbal STM, visual memory, auditory memory and the following language skills: expressive vocabulary, receptive vocabulary and syntactic production. The purpose of the present study is to investigate possible interrelation 
between linguistic and cognitive domains. We also explore whether memory (vSTM, immediate and delayed visual memory) and non-verbal IQ skills can predict language skills and vice versa. Since memory has been shown to contribute to higher cognitive functions and to be associated with language development in typical and atypical populations, then discovering a relationship between STM or visual memory in ASD could potentially shed some light on how best to communicate information to these children and suggest beneficial remedial interventions.

The specific questions the present study addresses are the following:

1) Is there a relationship between cognitive (non-verbal IQ, vSTM, immediate and delayed visual memory, visual information recall) and language skills (expressive vocabulary, receptive vocabulary, syntactic production) for children with ASD and which cognitive skills are strongly related with language skills?

2) To what extent can cognitive skills (non-verbal IQ, vSTM, immediate and delayed visual memory, visual information recall) predict the language skills (expressive vocabulary, receptive vocabulary, syntactic production) of school-aged children with ASD and to what extent can language skills predict their cognitive skills?

3) Do children with ASD with low cognitive skills always perform poorly on language tasks and vice versa?

\section{Method}

\subsection{Participants}

Thirty-three children ( 25 males; 8 females) 6 - 12 years old with ASD (with typical and low non-verbal IQ: range $30-130$ in RPM) were recruited from public primary schools (both mainstream and special education schools) in the city of Thessaloniki and in Peloponnese, Greece during autumn and winter of 2017-2018. Only those children whose parents gave written permission to participate in the research were included in the study and research ethics protocols were followed. From this cohort 18 children ( 13 males, 5 females; average age: 9; 5 years) had typical non-verbal IQ ( $>80$ in Raven Progressive Matrices) and 15 children (12 males, 3 females; average age: 8 ; 5 years) had low non-verbal IQ ( $<60$ in RPM). All children were diagnosed with ASD by public hospitals and public medical-pedagogical centres according to the DSM-V [1] and ICD-10 [60] official criteria.

\subsection{Procedure}

Participants were assessed at their school individually in one or two sessions of a total duration of 45 minutes.

\subsection{Tasks}

In this study, we employed tasks to assess cognitive as well language skills. They were all Greek standardised tests. More specifically, experimental tasks included: 


\subsubsection{Cognitive Measures}

Non-verbal IQ. Non-verbal IQ was assessed with the Greek version of Raven Standard Progressive Matrices [61]. Both standard scores and percentiles were taken into consideration.

Verbal short-term memory, visual and auditory memory. There were totally seven measures. Verbal short-term memory (vSTM) assessment included word recall, while visual and auditory memory included immediate and delayed visual memory and visual information recall. All measures were subtests of the Memory Test [62].

VSTM word recall was assessed using the Memory Test [62]. The child had to repeat a list of everyday words (not necessarily in the right order) and had five trials. Before every trial the list of words was presented to the child orally. For children 6 - 8 years old the list contained five words to be repeated, while for children 9 - 12 years old the list contained seven words. The score was calculated based on how many words the child recalled in every trial and how many trials it took him/her to remember all items of the list.

Immediate visual memory was assessed using a frame with 12 squares, in which the examiner placed chips in specific positions and then the child had to reproduce the same design. For children $6-8$ years old there were five chips, while for those $9-12$ years old there were seven chips to be placed in the correct position. There were five trials in total and before every trial the correct design was shown to the child. The score was calculated based on how many chips the child placed correctly in every trial and how many trials it took him/her to place all chips correctly. Delayed visual memory was assessed using the immediate visual memory subtest after a short break. The child had to remember the design s/he had learned before. There was only one trial and the score was calculated based on the number of the chips the child placed correctly. Visual information recall was assessed similarly to the immediate visual memory subtest, only this time the frame had images and there were two different colors of chips (red and yellow), five chips for children aged 6 - 8 years old and seven for children 9 - 12 years old respectively. Again there were five trials and the score was calculated based on how many chips of the right color the child placed correctly in every trial and how many trials it took him/her to place all chips correctly.

\subsubsection{Language Measures}

Expressive vocabulary. Expressive vocabulary (definitions) was assessed using the Greek version of Crichton Vocabulary Scales [63]. It contains 80 word definitions, presented orally, and arranged in order of increasing difficulty. The interruption criterion is four consecutive errors. Standard scores and percentiles were taken into consideration. Only one child among children with low non-verbal IQ skills was able to name a few definitions, so for all the rest of the children with low non-verbal IQ skills, picture naming and comprehension subscale were administered (see below).

Receptive Vocabulary. This task was administered only to children with low 
non-verbal IQ skills, because they couldn't be assessed with CVS, due to the fact that they were minimally verbal. Receptive vocabulary was assessed using Picture Naming and Comprehension Subscale from Detection of Speech and Language Disorders Test Preschool (DSLD Test, [64]). At first, the child was administered with Picture Naming test in which s/he was shown a picture from everyday life (e.g. fork) and s/he had to name it. When there were 10 consecutive errors, then Picture Comprehension test was administered in which the examiner would show 4 pictures (and ask the child to point to one of them).

Syntactic production: In accordance with Klem, Melby-Lervag, Hagtvet, Lyster, Gustafsson, and Hulme [65], we consider sentence recall (or sentence repetition) as a measure of syntactic production abilities. Sentence recall was a subtest of the TAAC [66], in which the child had to repeat the sentence s/he heard orally by the examiner. For each correct repetition of the sentence the child was given two points, one if there was one error and zero if there were two or more errors. The interruption criterion was two consecutive zeros.

\section{Results}

\subsection{Correlation Analysis}

In order to explore whether there is a relationship between cognitive and language skills and which cognitive skills are strongly related with language skills, non-parametric correlations (Spearman's rho) were conducted between measures of IQ, language and memory tasks (see Table 1 ).

Non-verbal IQ correlated significantly and strongly with all three language tasks (expressive vocabulary: $\mathrm{r}(33)=0.721, p<0.001$; receptive vocabulary: $\mathrm{r}(14)=0.59, p=0.025$; syntactic production: $\mathrm{r}(33)=0.067, p<0.001)$. VSTM word recall correlated strongly with expressive vocabulary $(\mathrm{r}(33)=0.88, p<0.001)$ and syntactic production $(\mathrm{r}(33)=0.87, p<0.001)$. Immediate visual memory correlated strongly with expressive vocabulary and syntactic production $(\mathrm{r}(33)=$ $0.72, p<0.001$ for both) and moderately, but not significantly, with receptive

Table 1. Non parametric correlations (Spearman's rho) between measures of non verbal IQ, language and memory.

\begin{tabular}{lcccccccc}
\hline & 1 & $\mathbf{2}$ & $\mathbf{3}$ & $\mathbf{4}$ & $\mathbf{5}$ & $\mathbf{6}$ & $\mathbf{7}$ & $\mathbf{8}$ \\
\hline 1) Non verbal IQ & 1 & & & & & & & \\
2) Expressive Vocabulary & $0.72^{* *}$ & 1 & & & & & & \\
3) Receptive Vocabulary & $0.62^{* *}$ & - & 1 & & & & \\
4) Syntactic Production & $0.66^{* *}$ & $0.91^{* *}$ & - & 1 & & & & \\
5) VSTM (word recall) & $0.76^{* *}$ & $0.88^{* *}$ & - & $0.87^{* *}$ & 1 & & \\
6) Immediate Visual Memory & $0.77^{* *}$ & $0.72^{* *}$ & 0.51 & $0.72^{* *}$ & $0.80^{* *}$ & 1 & & \\
7) Delayed Visual Memory & $0.67^{* *}$ & $0.78^{* *}$ & -0.05 & $0.75^{* *}$ & $0.78^{* *}$ & $0.82^{* *}$ & 1 & \\
8) Visual Information Recall & $0.67^{* *}$ & $0.71^{* *}$ & 0.32 & $0.73^{* *}$ & $0.79^{* *}$ & $0.92^{* *}$ & $0.90^{* *}$ & 1 \\
\hline
\end{tabular}

Note: ${ }^{*} p<0.05,{ }^{* *} p<0.001$. 
vocabulary $(\mathrm{r}(14)=0.51, p=0.62)$, while delayed visual memory correlated strongly with expressive vocabulary $(\mathrm{r}(33)=0.78, p<0.001)$ and with syntactic production $(\mathrm{r}(33)=0.75, p<0.001)$. Visual information recall correlated strongly with expressive vocabulary $(\mathrm{r}(33)=0.71, p<0.001)$ and syntactic production $(\mathrm{r}(33)=0.73, p<0.001)$.

\subsection{Regression Analysis}

\subsubsection{Can Performance on Cognitive Skills (Non-Verbal IQ, Memory) Predict the Language Skills (Expressive and Receptive Vocabulary, Syntactic Production) and Vice Versa?}

According to our research questions, we were first interested in exploring which cognitive skills (non-verbal IQ, vSTM, immediate and delayed visual memory, visual information recall) could better predict the language skills of school-aged children with ASD and more specifically expressive and receptive vocabulary and syntactic production. We thus performed three linear regression analyses with the stepwise method with expressive vocabulary, receptive vocabulary and syntactic production (all language measures) as the dependent variable (one at a time). The independent variables were non-verbal IQ, vSTM, visual memory (immediate and delayed) and visual information recall (see Table 2 and Figure 1). The results showed that expressive vocabulary was predicted significantly and strongly by vSTM and non-verbal IQ (vSTM only: $\mathrm{R}^{2}=0.83$, both vSTM \& non-verbal IQ: $\left.\mathrm{R}^{2}=0.88\right)$, syntactic production was predicted significantly by $\operatorname{vSTM}\left(\mathrm{R}^{2}=0.34\right)$ and receptive vocabulary was predicted significantly by non-verbal IQ $\left(\mathrm{R}^{2}=0.45\right)$.

Conversely, we were interested in exploring which language skills (expressive vocabulary, syntactic production) could better predict the cognitive skills (non-verbal IQ, vSTM, immediate and delayed visual memory and visual information recall) of children with ASD. We thus performed five linear regression analyses with the stepwise method with non-verbal IQ, vSTM, immediate and

Table 2. Results of linear regression analyses: effect of cognitive skills (non-verbal IQ, vSTM, immediate and delayed visual memory and visual information recall) on language skills (expressive vocabulary, syntactic production, receptive vocabulary respectively).

\begin{tabular}{ccccccc}
\hline Category & Variable & $\mathrm{B}$ & $\mathrm{SE}$ & $\mathrm{t}$ & $p$ & $\mathrm{R}^{2}$ \\
\hline Expressive vocabulary & (Constant) & 7.05 & 4.13 & 1.71 & 0.098 & 0.83 \\
& vSTM & 3.10 & 0.26 & 12.12 & 0.000 & \\
& (Constant) & -13.67 & 6.50 & -2.10 & 0.044 & 0.88 \\
& vSTM & 2.15 & 0.33 & 6.47 & 0.000 & \\
& Non verbal IQ & 0.44 & 0.12 & 3.76 & 0.001 & \\
Syntactic production & (Constant) & 0.23 & 2.67 & 0.09 & 0.932 & 0.34 \\
& vSTM & 0.65 & 0.17 & 3.96 & 0.000 & \\
Receptive vocabulary & (Constant) & -3.83 & 2.13 & -180 & 0.097 & 0.45 \\
& Non verbal IQ & 0.16 & 0.05 & 3.14 & 0.008 & \\
\hline
\end{tabular}




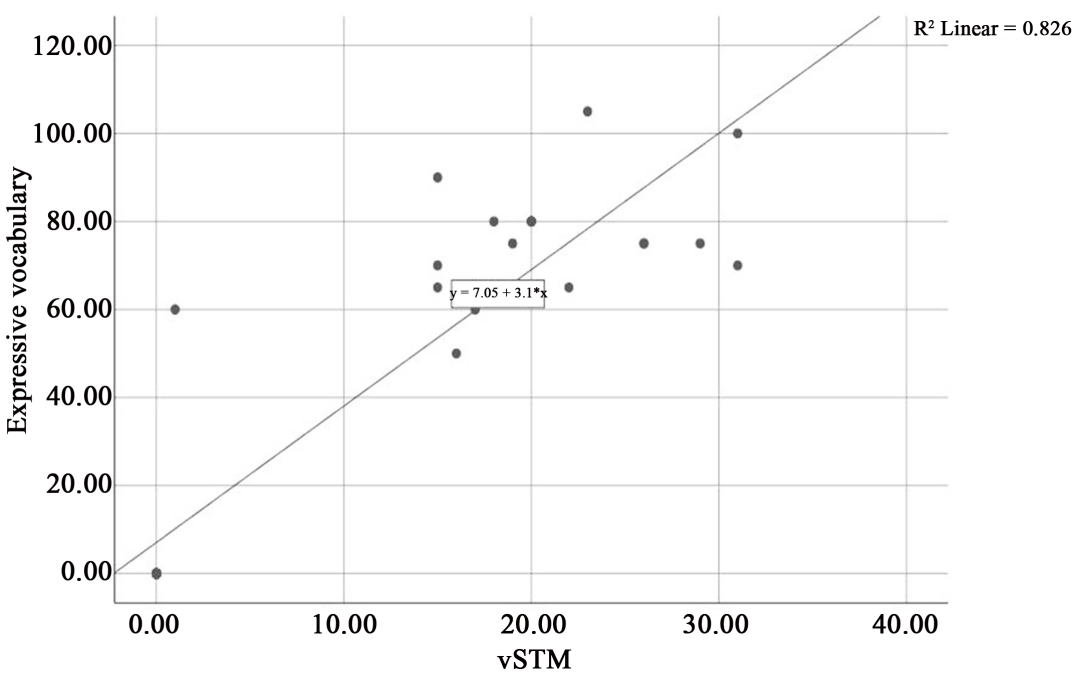

(a)

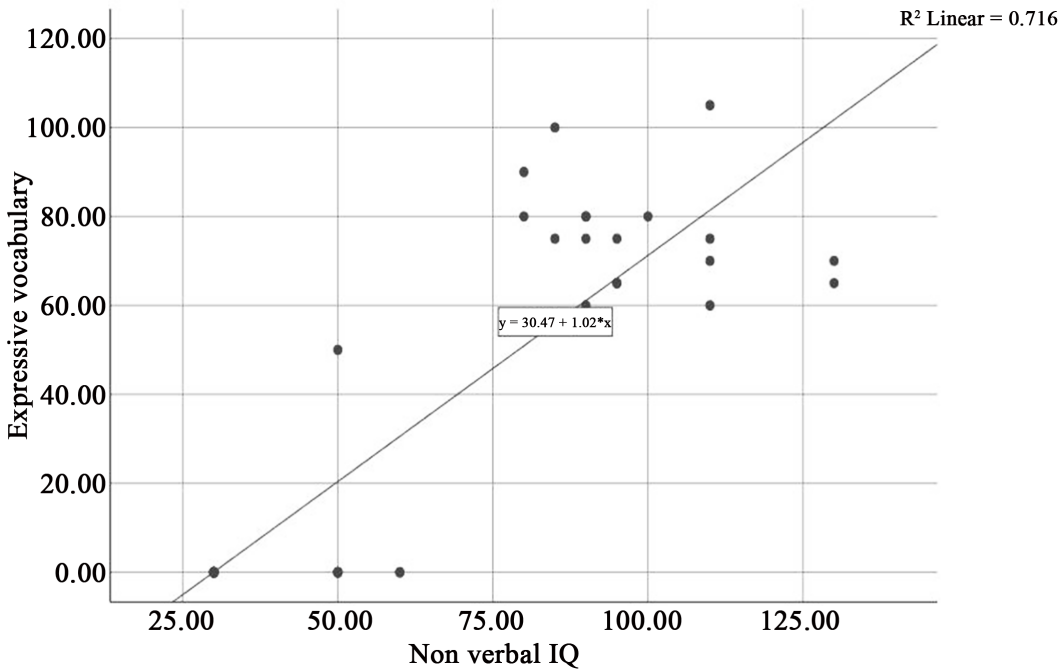

(b)

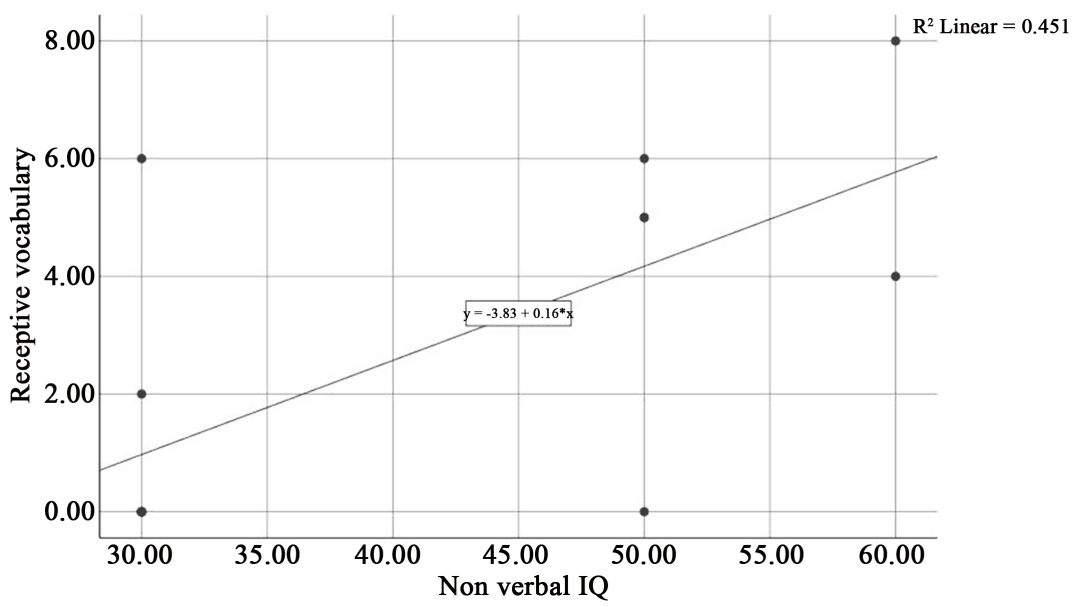

(c)

Figure 1. Scatterplots from the regression analyses (effect of cognitive skills on language skills): (a) effect of vSTM on expressive vocabulary; (b) effect of non verbal IQ on expressive vocabulary and (c) effect of non verbal IQ on receptive vocabulary. 
delayed visual memory and visual information recall as the dependent variable (one at a time) and expressive vocabulary and syntactic production as the independent variables (see Table 3 and Figure 2). The results showed that expressive vocabulary could predict significantly and strongly non-verbal IQ $\left(\mathrm{R}^{2}=0.72\right)$, $\operatorname{vSTM}\left(R^{2}=0.83\right)$, immediate visual memory $\left(R^{2}=0.62\right)$, delayed visual memory $\left(\mathrm{R}^{2}=0.54\right)$, and -significantly but not strongly-visual information recall $\left(\mathrm{R}^{2}=\right.$ $0.44)$.

\subsubsection{Summary of the Regression Analysis Findings}

In sum, as it can be seen in Table $2 \&$ Table 3 and Figure $1 \&$ Figure 2, on the one hand VSTM was found to be a significant predictor for expressive and receptive vocabulary and syntactic production, while on the other hand expressive vocabulary was found to be a significant predictor for all cognitive measures: non-verbal IQ, vSTM, immediate and delayed visual memory and visual information recall.

\subsection{Individual Analysis}

Individual data analysis was conducted for children with ASD, to investigate whether children with ASD with low cognitive skills (non-verbal IQ, memory) always perform poorly on language tasks, and vice versa. In addition, we classified the results following a Guttman scale (or cumulative scale). Individual data are presented in the Appendix.

Thirty out of thirty three children with ASD had a below mean TD performance in either cognitive (non-verbal IQ, vSTM, immediate and delayed visual memory, visual information recall) or language measures (expressive and receptive vocabulary, syntactic production). Twenty-four had low performance in both cognitive and language measures and six had a low performance only in at least one cognitive measure. Finally, there were 3 children with ASD who had average or above average performance in both cognitive and language domains.

Table 3. Results of linear regression analyses: effect of language skills (expressive vocabulary, syntactic production) on cognitive skills (non-verbal IQ, vSTM, immediate and delayed visual memory and visual information recall respectively).

\begin{tabular}{ccccccc}
\hline \multirow{2}{*}{ Category } & Variable & $\mathrm{B}$ & Std. Error & $\mathrm{t}$ & $p$ & $\mathrm{R}^{2}$ \\
\hline Non-verbal IQ & (Constant) & 41.96 & 4.58 & 9.16 & 0.000 & 0.72 \\
vSTM & Expressive vocabulary & 0.70 & 0.08 & 8.85 & 0.000 & \\
& (Constant) & 0.14 & 1.27 & 0.11 & 0.910 & 0.83 \\
& Expressive vocabulary & 0.27 & 0.02 & 12.12 & 0.000 & \\
Immediate visual memory & (Constant) & 3.85 & 2.14 & 1.80 & 0.082 & 0.62 \\
& Expressive vocabulary & 0.26 & 0.04 & 7.10 & 0.000 & \\
Delayed visual memory & (Constant) & 0.36 & 0.47 & 0.78 & 0.444 & 0.54 \\
& Expressive vocabulary & 0.05 & 0.01 & 6.02 & 0.000 \\
Visual information recall & (Constant) & 0.25 & 0.89 & 0.28 & 0.779 & 0.44 \\
& Expressive vocabulary & 0.08 & 0.02 & 4.93 & 0.000 \\
\hline
\end{tabular}




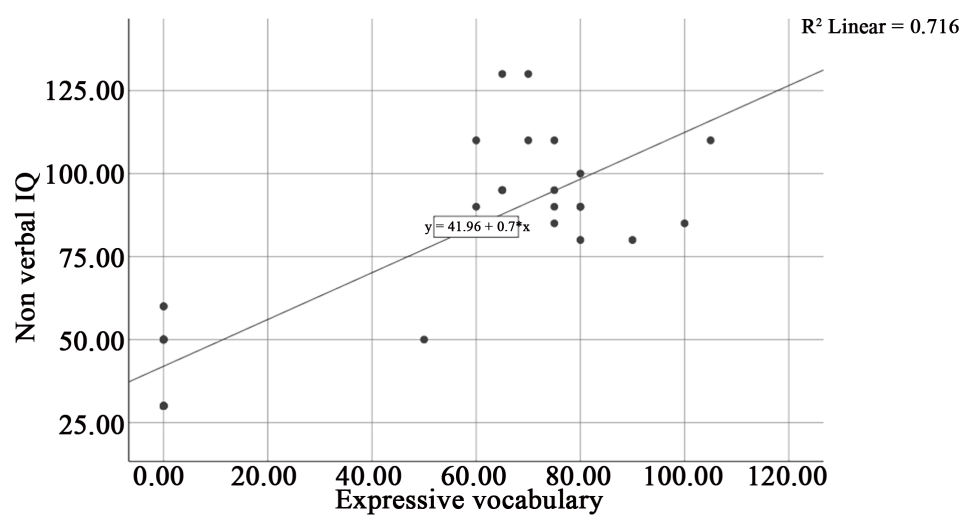

(a)

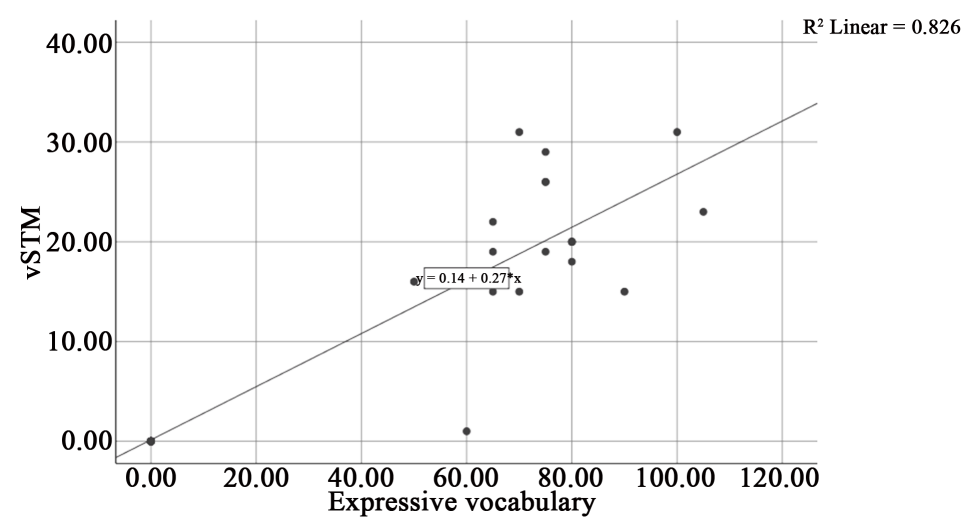

(b)

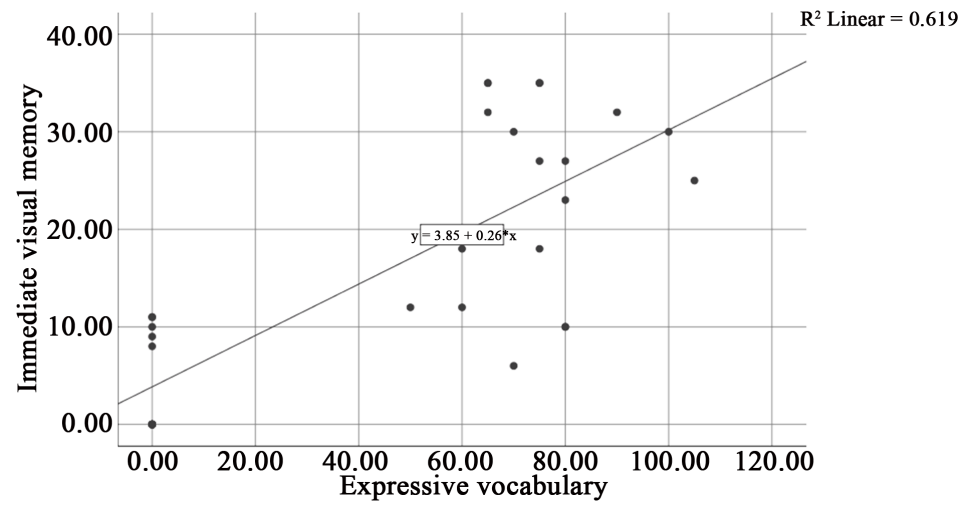

(c)

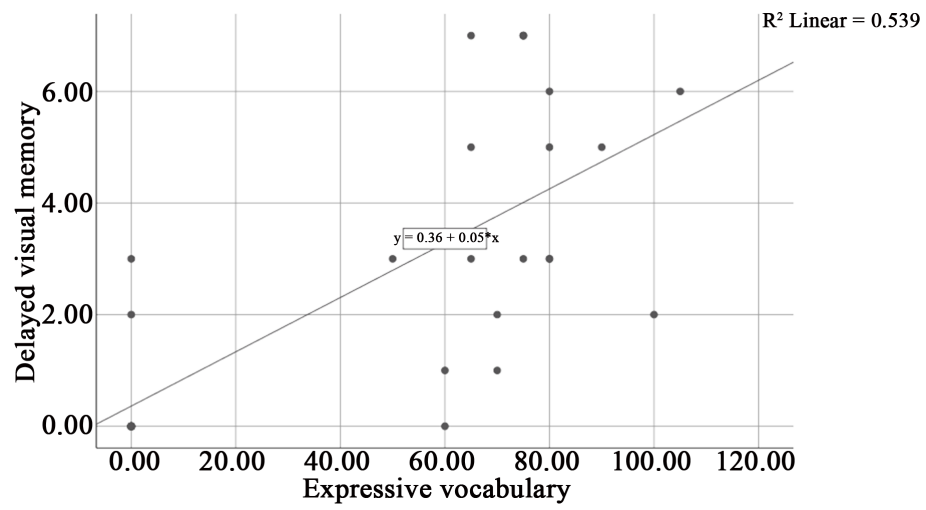

(d) 


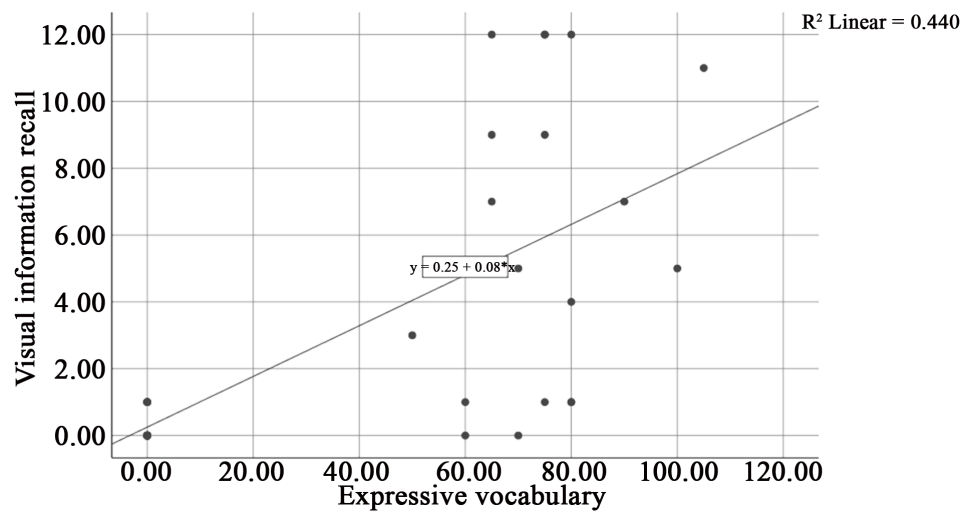

(e)

Figure 2. Scatterplots from the regression analyses (effect of language skills on cognitive skills): (a) effect of expressive vocabulary on non verbal IQ; (b) effect of expressive vocabulary on vSTM; (c) effect of immediate visual memory on expressive vocabulary; (d) effect of expressive vocabulary on delayed visual memory; (e) effect of expressive vocabulary on visual information recall.

An interesting point was that for two children delayed visual memory was intact, while immediate visual memory was impaired. The opposite, which was more expected, happened for five children. Moreover, four children had a low performance only in visual memory (immediate or delayed) and not in vSTM. In sum, the above findings show that limitations in non-verbal IQ and memory almost systematically co-occur, but they also show that highly selective limitations in these domains can exist in ASD.

\section{Discussion}

In the present study, we assessed school-aged children with ASD with typical and low verbal IQ on cognitive and language measures. The aim of this study was to investigate whether there is an interrelation between cognitive and language skills in ASD, since there is such a large heterogeneity in cognitive and linguistic profiles among ASD individuals and findings are controversial. We were also interested in exploring the role of memory in the cognitive function of individuals with ASD. More specifically, we evaluated which cognitive skills can predict the language skills (expressive and receptive vocabulary and syntactic production) of children with ASD and vice versa. Finally, we were interested in determining if children with ASD with low cognitive skills always perform poorly on language tasks and vice versa.

Correlations between cognitive and language measures indicated that nonverbal IQ correlated significantly and strongly with all three language tasks, while memory tasks (vSTM, immediate and delayed visual memory, visual information recall) correlated significantly and strongly with expressive vocabulary and syntactic production.

Furthermore, our study showed a vSTM-but not a visual memory-effect in linguistic abilities. More specifically, regression analysis showed that expressive 
vocabulary was predicted by non-verbal IQ and vSTM to a large extent, syntactic production was predicted by vSTM and picture comprehension was predicted by non-verbal IQ. Conversely, expressive vocabulary could predict significantly non-verbal IQ, vSTM, immediate and delayed visual memory and visual information recall. In this respect, we suggest that VSTM is a strong predictor of language skills for children with ASD, just like it is for other typical and atypical populations reported in literature [46] [47] [67] [68] [69].

Furthermore, individual analysis showed that dissociations exist between non-verbal IQ and memory on the one hand and language skills (expressive vocabulary, syntactic production) on the other hand. Thirty out of 33 children with ASD had a below mean TD performance in either cognitive (non-verbal IQ, vSTM, immediate and delayed visual memory, visual information recall) or language measures (expressive vocabulary, syntactic production, picture comprehension). Twenty-four had low performance in both cognitive and language measures and six had a low performance in at least one cognitive measure. In sum, the above findings show that low performance in non-verbal IQ and memory almost systematically co-occur with low performance in expressive vocabulary, syntactic production and picture comprehension for most but not all children with ASD.

\section{Limitations and Future Directions}

The results of the present study of cognitive and language skills of children with ASD must be interpreted with caution for the following reasons: The sample was relatively small with a wide age range and no generalizations can be made to all children with ASD. In order to develop adequate educational and language interventions, we have to fully understand the cognitive and language challenges of individuals with ASD. The information about such skills is also important to compare such performance to that of children with other developmental disabilities that also show difficulties in memory and language, such as ADHD, developmental language disorder and developmental dyslexia.

\section{Acknowledgements}

The author gratefully acknowledges the support of her supervisor Prof. Stavroula Stavrakaki, as well as the contribution of all children and their families. This post-doctoral research is co-financed by Greece and the European Union (European Social Fund, ESF) through the Operational Programme "Human Re-

sources Development, Education and Lifelong Learning" in the context of the project "Reinforcement of Postdoctoral Researchers" (MIS-5001552), implemented by the State Scholarships Foundation (IKY).

\section{Conflicts of Interest}

The authors declare no conflicts of interest regarding the publication of this paper. 


\section{References}

[1] American Psychiatric Association (2013) Diagnostic and Statistical Manual of Mental Disorders. 5th Edition, American Psychiatric Publishing, Arlington, VA.

[2] Baio, J., Wiggins, L., Christensen, D. L., Maenner, M. J., Daniels, J., Warren, Z. and Durkin, M. S. (2018) Prevalence of Autism Spectrum Disorder among Children Aged 8 Years-Autism and Developmental Disabilities monitoring Network, 11 Sites, United States, 2014. MMWR Surveillance Summaries, 67, 1. https://doi.org/10.15585/mmwr.ss6706a1

[3] Xu, G., Strathearn, L., Liu, B. and Bao, W. (2018) Prevalence of Autism Spectrum Disorder among US Children and Adolescents, 2014-2016. Jama, 319, 81-82. https://doi.org/10.1001/jama.2017.17812

[4] Hellendoorn, A., Wijnroks, L., Van Daalen, E., Dietz, C., Buitelaar, J.K. and Leseman, P. (2015) Motor Functioning, Exploration, Visuospatial Cognition and Language Development in Preschool Children with Autism. Research in Developmental Disabilities, 39, 32-42. https://doi.org/10.1016/j.ridd.2014.12.033

[5] Wang, Z., Jing, J., Igarashi, K., Fan, L., Yang, S., Li, Y. and Jin, Y. (2018) Executive Function Predicts the Visuospatial Working Memory in Autism Spectrum Disorder and Attention-Deficit/Hyperactivity Disorder. Autism Research, 11, 1148-1156. https://doi.org/10.1002/aur.1967

[6] Wang, Y., Zhang, Y.B., Liu, L.L., Cui, J.F., Wang, J., Shum, D.H. and Chan, R.C. (2017) A Meta-Analysis of Working Memory Impairments in Autism Spectrum Disorders. Neuropsychology Review, 27, 46-61. https://doi.org/10.1007/s11065-016-9336-y

[7] Barendse, E.M., Schreuder, L.J., Thoonen, G., Hendriks, M.P., Kessels, R.P., Backes, W.H. and Jansen, J.F. (2018) Working Memory Network Alterations in High-Functioning Adolescents with an Autism Spectrum Disorder. Psychiatry and Clinical Neurosciences, 72, 73-83. https://doi.org/10.1111/pcn.12602

[8] Bowler, D.M., Poirier, M., Martin, J.S. and Gaigg, S.B. (2016) Nonverbal Short-Term Serial Order Memory in Autism Spectrum Disorder. Journal of Abnormal Psychology, 125, 886-893. https://doi.org/10.1037/abn0000203

[9] Meir, N. and Novogrodsky, R. (2019) Syntactic Abilities and Verbal Memory in Monolingual and Bilingual Children with High Functioning Autism (HFA). First Language. https://doi.org/10.1177/0142723719849981

[10] Gernsbacher, M.A., Morson, E.M. and Grace, E.J. (2016) Language Development in Autism. In Neurobiology of Language, Academic Press, 879-886.

[11] Eigsti, I.M., de Marchena, A.B., Schuh, J.M. and Kelley, E. (2011) Language Acquisition in Autism Spectrum Disorders: A Developmental Review. Research in Autism Spectrum Disorders, 5, 681-691. https://doi.org/10.1016/j.rasd.2010.09.001

[12] Canfield, A.R., Eigsti, I.M., de Marchena, A. and Fein, D. (2016) Story Goodness in Adolescents with Autism Spectrum Disorder (ASD) and in Optimal Outcomes from ASD. Journal of Speech, Language, and Hearing Research, 59, 533-545. https://doi.org/10.1044/2015 JSLHR-L-15-0022

[13] Kelley, E., Paul, J.J., Fein, D. and Naigles, L.R. (2006) Residual Language Deficits in Optimal Outcome Children with a History of Autism. Journal of Autism and Developmental Disorders, 36, 807-828. https://doi.org/10.1007/s10803-006-0111-4

[14] Suh, J., Eigsti, I., Naigles, L., Barton, M., Kelley, E. and Fein, D. (2014) Narrative Performance of Optimal Outcome Children and Adolescents with a History of an Autism Spectrum Disorder (ASD). Journal of Autism and Developmental Disor- 
ders, 44, 1681-1694. https://doi.org/10.1007/s10803-014-2042-9

[15] Tek, S., Mesite, L., Fein, D. and Naigles, L. (2014) Longitudinal Analyses of Expressive Language Development Reveal Two Distinct Language Profiles among Young Children with Autism Spectrum Disorders. Journal of Autism and Developmental Disorders, 44, 75-89. https://doi.org/10.1007/s10803-013-1853-4

[16] Kjelgaard, M.M. and Tager-Flusberg, H. (2001) An Investigation of Language Impairment in Autism: Implications for Genetic Subgroups. Language and Cognitive Processes, 16, 287-308. https://doi.org/10.1080/01690960042000058

[17] Durrleman, S. and Delage, H. (2016) Autism Spectrum Disorder and Specific Language Impairment: Overlaps in Syntactic Profiles. Language Acquisition, 23, 361-386. https://doi.org/10.1080/10489223.2016.1179741

[18] Eigsti, I.M., Bennetto, L. and Dadlani, M.B. (2007) Beyond Pragmatics: Morphosyntactic Development in Autism. Journal of Autism and Developmental Disorders, 37, 1007-1023. https://doi.org/10.1007/s10803-006-0239-2

[19] Modyanova, N., Perovic, A. and Wexler, K. (2017) Grammar Is Differentially Impaired in Subgroups of Autism Spectrum Disorders: Evidence from an Investigation of Tense Marking and Morphosyntax. Frontiers in Psychology, 8, 320. https://doi.org/10.3389/fpsyg.2017.00320

[20] Kasari, C., Brady, N., Lord C. and Tager-Flusberg, H. (2013) Assessing the Minimally Verbal School-Aged Child with Autism Spectrum Disorder. Autism Research, 6, 479-493. https://doi.org/10.1002/aur.1334

[21] Ellis Weismer, S., Davidson, M.M., Gangopadhyay, I., Sindberg, H., Roebuck, H. and Kaushanskaya, M. (2017) The Role of Nonverbal Working Memory in Morphosyntactic Processing by Children with Specific Language Impairment and Autism Spectrum Disorders. Journal of Neurodevelopmental Disorders, 9, 28. https://doi.org/10.1186/s11689-017-9209-6

[22] Lloyd, V., Gatherer, A. and Kalsy, S. (2006) Conducting Qualitative Interview Research with People with Expressive Language Difficulties. Qualitative Health Research, 16, 1386-1404. https://doi.org/10.1177/1049732306293846

[23] Sukenik, N. and Friedmann, N. (2018) ASD Is Not DLI: Individuals with Autism and Individuals with Syntactic DLI Show Similar Performance Level in Syntactic Tasks, but Different Error Patterns. Frontiers in Psychology, 9, 279. https://doi.org/10.3389/fpsyg.2018.00279

[24] Jensen de López, K., Schroeder, K. and Gavarró, A. (2018) Successful Passive Sentence Comprehension among Danish Adolescents with Autism Spectrum Disorders. Autism \& Developmental Language Impairments, 3, 1-16. https://doi.org/10.1177/2396941518761239

[25] Joseph, R.M., Tager-Flusberg, H. and Lord, C. (2002) Cognitive Profiles and SocialCommunicative Functioning in Children with Autism Spectrum Disorder. Journal of Child Psychology and Psychiatry, 43, 807-821.

https://doi.org/10.1111/1469-7610.00092

[26] Terzi, A., Marinis, T., Kotsopoulou, A. and Francis, K. (2014) Grammatical Abilities of Greek-Speaking Children with Autism. Language Acquisition, 21, 4-44. https://doi.org/10.1080/10489223.2013.855216

[27] Terzi, A., Zafeiri, A., Marinis, T. and Francis, K. (2017) Object Clitics in the Narratives of High-Functioning Children with Autism. Proceedings of Boston University Conference on Language Development, 41, 637-650.

[28] Mayes, S.D. and Calhoun, S.L. (2003) Ability Profiles in Children with Autism: Influence of Age and IQ. Autism, 7, 65-80. 
https://doi.org/10.1177/1362361303007001006

[29] Fein, D., Stevens, M., Dunn, M., Waterhouse, L., Allen, D., Rapin, I. and Feinstein, C. (1999) Subtypes of Pervasive Developmental Disorder: Clinical Characteristics. Child Neuropsychology, 5, 1-23. https://doi.org/10.1076/chin.5.1.1.7075

[30] Grondhuis, S.N., Lecavalier, L., Arnold, L.E., Handen, B.L., Scahill, L., McDougle, C.J. and Aman, M.G. (2018) Differences in Verbal and Nonverbal IQ Test Scores in Children with Autism Spectrum Disorder. Research in Autism Spectrum Disorders, 49, 47-55. https://doi.org/10.1016/j.rasd.2018.02.001

[31] Boucher, J. and Warrington, E.K. (1976) Memory Deficits in Early Infantile Autism: Some Similarities to the Amnesic Syndrome. British Journal of Psychology, 67, 73-87. https://doi.org/10.1111/j.2044-8295.1976.tb01499.x

[32] Hermelin, B. and O’Connor, N. (1970) Psychological Experiments with Autistic Children. Pergamon Press, Oxford, UK.

[33] Williams, D., Happé, F. and Jarrold, C. (2008) Intact Inner Speech Use in Autism Spectrum Disorder: Evidence from a Short-Term Memory Task. Journal of Child Psychology and Psychiatry, 49, 51-58. https://doi.org/10.1111/j.1469-7610.2007.01836.x

[34] Boucher, J. and Bowler, D.M. (2008) Memory in Autism: Theory and Evidence. Cambridge University Press, Cambridge.

[35] Gaigg, S.B., Bowler, D.M. and Gardiner, J.M. (2014) Episodic but Not Semantic Order Memory Difficulties in Autism Spectrum Disorder: Evidence from the Historical Figures Task. Memory, 22, 669-678. https://doi.org/10.1080/09658211.2013.811256

[36] Poirier, M., Martin, J.S., Gaigg, S.B. and Bowler, D.M. (2011) Short-Term Memory in Autism Spectrum Disorder. Journal of abnormal psychology, 120, 247-252. https://doi.org/10.1037/a0022298

[37] Kercood, S., Grskovic, J.A., Banda, D. and Begeske, J. (2014) Working Memory and Autism: A Review of Literature. Research in Autism Spectrum Disorders, 8, 1316-1332. https://doi.org/10.1016/j.rasd.2014.06.011

[38] Chien, Y.L., Gau, S.F., Shang, C.Y., Chiu, Y.N., Tsai, W.C. and Wu, Y.Y. (2015) Visual Memory and Sustained Attention Impairment in Youths with Autism Spectrum Disorders. Psychological Medicine, 45, 2263-2273. https://doi.org/10.1017/S0033291714003201

[39] Ameli, R., Courchesne, E., Lincoln, A., Kaufman, A.S. and Grillon, C. (1988) Visual Memory Processes in High-Functioning Individuals with Autism. Journal of Autism and Developmental Disorders, 18, 601-615. https://doi.org/10.1007/BF02211878

[40] Salmanian, M., Tehrani-Doost, M., Ghanbari-Motlagh, M. and Shahrivar, Z. (2012) Visual Memory of Meaningless Shapes in Children and Adolescents with Autism Spectrum Disorders. Iran Journal of Psychiatry, 7, 104-108.

[41] Funabiki, Y. and Shiwa, T. (2018) Weakness of Visual Working Memory in Autism. Autism Research, 11, 1245-1252. https://doi.org/10.1002/aur.1981

[42] Baddeley, A. (2007) Working Memory, Thought, and Action (Vol. 45). OUP Oxford.

[43] Cowan, N. (2016) Working Memory Capacity: Classic Edition. Routledge.

[44] de Abreu, P.M.J.E., Gathercole, S.E. and Martin, R. (2011) Disentangling the Relationship between Working Memory and Language: The Roles of Short-Term Storage and Cognitive Control. Learning and Individual Differences, 21, 569-574. 
https://doi.org/10.1016/j.lindif.2011.06.002

[45] Gathercole, S.E. and Baddeley, A.D. (2014) Working Memory and Language. Psychology Press, England.

[46] Gupta, P. (2003) Examining the Relationship between Word Learning, Nonword Repetition and Immediate Serial Recall in Adults. The Quarterly Journal of Experimental Psychology, 56A, 1213-1236. https://doi.org/10.1080/02724980343000071

[47] Gupta, P. and MacWhinney, B. (1997) Vocabulary Acquisition and Verbal ShortTerm Memory: Computational and Neural Bases. Brain and language, 59, 267-333. https://doi.org/10.1006/brln.1997.1819

[48] Hick, R.F., Botting, N. and Conti-Ramsden, G. (2005) Short-Term Memory and Vocabulary Development in Children with Down Syndrome and Children with Specific Language Impairment. Developmental Medicine and Child Neurology, 47, 532-538. https://doi.org/10.1017/S0012162205001040

[49] Majerus, S., Poncelet, M., Greffe, C. and Van der Linden, M. (2006) Relations between Vocabulary Development and Verbal Short-Term Memory: The Relative Importance of Short-Term Memory for Serial Order and Item Information. Journal of Experimental Child Psychology, 93, 95-119.

https://doi.org/10.1016/j.jecp.2005.07.005

[50] Pisoni, D., Kronenberger, W., Roman, A. and Geers, A. (2011) Article 7: Measures of Digit Span and Verbal Rehearsal Speed in Deaf Children Following More than 10 Years of Cochlear Implantation. Ear and Hearing, 32, 60. https://doi.org/10.1097/AUD.0b013e3181ffd58e

[51] Faja, S. and Dawson, G. (2014) Performance on the Dimensional Change Card Sort and Backward Digit Span by Young Children with Autism without Intellectual Disability. Child Neuropsychology, 20, 692-699. https://doi.org/10.1080/09297049.2013.856395

[52] Phelan, H.L., Filliter, J.H. and Johnson, S.A. (2011) Brief Report: Memory Performance on the California Verbal Learning Test: Children's Version in Autism Spectrum Disorder. Journal of Autism and Developmental Disorders, 41, 518-523. https://doi.org/10.1007/s10803-010-1069-9

[53] Gabig, C.S. (2008) Verbal Working Memory and Story Retelling in School-Age Children with Autism. Language, Speech, and Hearing Services in Schools, 39, 498-511. https://doi.org/10.1044/0161-1461(2008/07-0023)

[54] Durrleman, S., Delage, H., Tuller, L. and Prévost, P. (2017) The Comprehension of Passives in Autism Spectrum Disorder. Glossa: A Journal of General Linguistics, 2, 88. https://doi.org/10.5334/gjgl.205

[55] Tyson, K., Kelley, E., Fein, D., Orinstein, A., Troyb, E., Barton, M. and Helt, M. (2014) Language and Verbal Memory in Individuals with a History of Autism Spectrum Disorders who Have Achieved Optimal Outcomes. Journal of Autism and Developmental Disorders, 44, 648-663. https://doi.org/10.1007/s10803-013-1921-9

[56] Lindgren, K.A., Folstein, S.E., Tomblin, J.B. and Tager-Flusberg, H. (2009) Language and Reading Abilities of Children with Autism Spectrum Disorders and Specific Language Impairment and Their First-Degree Relatives. Autism Research, 2, 22-38. https://doi.org/10.1002/aur.63

[57] Hill, A.P., Van Santen, J., Gorman, K., Langhorst, B.H. and Fombonne, E. (2015) Memory in Language-Impaired Children with and without Autism. Journal of Neurodevelopmental Disorders, 7, 19. https://doi.org/10.1186/s11689-015-9111-z

[58] Taylor, L.J., Maybery, M.T., Grayndler, L. and Whitehouse, A.J. (2014) Evidence for Distinct Cognitive Profiles in Autism Spectrum Disorders and Specific Language 
Impairment. Journal of Autism and Developmental Disorders, 44, 19-30. https://doi.org/10.1007/s10803-013-1847-2

[59] Williams, D., Payne, H. and Marshall, C. (2013) Non-Word Repetition Impairment in Autism and Specific Language Impairment: Evidence for Distinct Underlying Cognitive Causes. Journal of Autism and Developmental Disorders, 43, 404-417. https://doi.org/10.1007/s10803-012-1579-8

[60] World Health Organization (2004) ICD-10: International Statistical Classification of Diseases and Related Health Problems: Tenth Revision, 2nd Edition.

[61] Sideridis, G.D., Antoniou, F., Mouzaki, A. and Simos, P. (2015) The Greek version of Raven's Colored Progressive Matrices. Motivo Assessment, Athens.

[62] Bezevegis, E., Economou, A. and Milonas, K. (2007) Memory Test. Hellenic Ministry of Education-EPEAEK, Athens.

[63] Sideridis, G.D., Antoniou, F., Mouzaki, A. and Simos, P. (2015) The Greek Version of Crichton Vocabulary Scale. Motivo Assessment, Athens.

[64] Oikonomou, A., Bezevegis, I., Milonas, K. and Varlokosta, S. (2007) Screening Tool for the Detection of Speech and Language Disorders for Preschoolers. Hellenic Ministry of Education-EPEAEK, Athens.

[65] Klem, M., Melby-Lervåg, M., Hagtvet, B., Lyster, S. A.H., Gustafsson, J.E. and Hulme, C. (2015) Sentence Repetition Is a Measure of Children's Language Skills Rather than Working Memory Limitations. Developmental Science, 18, 146-154. https://doi.org/10.1111/desc.12202

[66] Simos, P. Mouzaki, A. and Sideridis, G. (2007) Test for the Assessment of Attention and Concentration. Hellenic Ministry of Education-EPEAEK, Athens.

[67] Archibald, L.M. and Gathercole, S.E. (2006) Short-Term and Working Memory in Specific Language Impairment. International Journal of Language \& Communication Disorders, 41, 675-693. https://doi.org/10.1080/13682820500442602

[68] Leclercq, A.L. and Majerus, S. (2010) Serial-Order Short-Term Memory Predicts Vocabulary Development: Evidence from a Longitudinal Study. Developmental Psychology, 46, 417-427. https://doi.org/10.1037/a0018540

[69] Næss, K.A.B., Lyster, S.A.H., Hulme, C. and Melby-Lervåg, M. (2011) Language and Verbal Short-Term Memory Skills in Children with Down Syndrome: A Meta-Analytic Review. Research in Developmental Disabilities, 32, 2225-2234. https://doi.org/10.1016/j.ridd.2011.05.014 


\section{Appendix}

Table A1. Individual data of children with ASD on cognitive and language measures (dark grey indicates double deficit in memory and language skills, while light grey indicates deficit only in language or memory skills).

\begin{tabular}{|c|c|c|c|c|c|c|c|c|}
\hline Subjects & $\begin{array}{c}\text { Picture } \\
\text { comprehension }\end{array}$ & $\begin{array}{c}\text { Non-verbal } \\
\text { IQ }\end{array}$ & $\begin{array}{l}\text { Expressive } \\
\text { vocabulary }\end{array}$ & vSTM & $\begin{array}{c}\text { Immediate } \\
\text { visual memory }\end{array}$ & $\begin{array}{c}\text { Delayed } \\
\text { visual memory }\end{array}$ & $\begin{array}{l}\text { Syntactic } \\
\text { production }\end{array}$ & $\begin{array}{c}\text { Visual } \\
\text { information recall }\end{array}$ \\
\hline ASD1 & & 95 & 75 & 26 & 27 & 20 & 7 & 9 \\
\hline ASD17 & & 110 & 75 & 26 & 35 & 20 & 7 & 12 \\
\hline ASD21 & & 100 & 80 & 20 & 23 & 40 & 5 & 12 \\
\hline ASD2 & & 80 & 90 & $15^{*}$ & 32 & 20 & 5 & 7 \\
\hline ASD3 & & 130 & 65 & 19 & 35 & $1^{*}$ & 7 & 12 \\
\hline ASD4 & & 90 & 80 & 18 & 27 & 20 & 6 & $4^{*}$ \\
\hline ASD18 & & 85 & 75 & 29 & 35 & $1^{*}$ & 7 & 12 \\
\hline ASD32 & & 110 & 105 & 23 & 25 & $10^{*}$ & 6 & 11 \\
\hline ASD13 & & 95 & 65 & 22 & 35 & $1^{*}$ & $3^{*}$ & 9 \\
\hline ASD20 & & 110 & 70 & 31 & 30 & 20 & $2^{*}$ & $5^{*}$ \\
\hline ASD24 & & 95 & 65 & $15^{*}$ & 32 & $1^{\star}$ & 5 & 7 \\
\hline ASD33 & & 85 & 100 & 31 & 30 & 20 & $2^{*}$ & $5^{*}$ \\
\hline ASD15 & & 80 & 80 & 20 & $10^{*}$ & 40 & $3^{*}$ & $1^{*}$ \\
\hline ASD27 & & 90 & 80 & 20 & $10^{*}$ & 40 & $3^{*}$ & $1^{*}$ \\
\hline ASD28 & & 90 & 75 & 19 & $18^{*}$ & $1^{*}$ & $3^{*}$ & $1^{*}$ \\
\hline ASD16 & & 110 & 75 & $1^{*}$ & $12^{*}$ & $1^{*}$ & $0^{*}$ & $0^{*}$ \\
\hline ASD19 & & 130 & 70 & $15^{*}$ & $6^{*}$ & $0^{*}$ & $1^{*}$ & $0^{*}$ \\
\hline ASD26 & & 90 & 60 & $17^{*}$ & $18^{*}$ & $1^{*}$ & $1^{*}$ & $1^{*}$ \\
\hline ASD30 & & $50^{*}$ & $50^{*}$ & $16^{*}$ & $12^{*}$ & $1^{\star}$ & $3^{*}$ & $3^{*}$ \\
\hline ASD5 & $0^{*}$ & $50^{*}$ & $0^{*}$ & $0^{*}$ & $9^{*}$ & $0^{*}$ & $3^{*}$ & $1^{*}$ \\
\hline ASD6 & $4^{*}$ & $60^{*}$ & $0^{*}$ & $0^{*}$ & $11^{*}$ & $0^{*}$ & $0^{*}$ & $1^{*}$ \\
\hline ASD7 & $5^{*}$ & $50^{*}$ & $0^{*}$ & $0^{*}$ & $10^{*}$ & $0^{*}$ & $2^{*}$ & $1^{*}$ \\
\hline ASD8 & $2^{*}$ & $30^{*}$ & $0^{*}$ & $0^{*}$ & $0^{*}$ & $0^{*}$ & $0^{*}$ & $0^{*}$ \\
\hline ASD9 & $8^{*}$ & $60^{*}$ & $0^{*}$ & $0^{*}$ & $11^{*}$ & $0^{*}$ & $0^{*}$ & $1^{*}$ \\
\hline ASD10 & $6^{*}$ & $50^{*}$ & $0^{*}$ & $0^{*}$ & $8^{*}$ & $0^{*}$ & $0^{*}$ & $0^{*}$ \\
\hline ASD11 & $5^{*}$ & $50^{*}$ & $0^{*}$ & $0^{*}$ & $0^{*}$ & $0^{*}$ & $0^{*}$ & $0^{*}$ \\
\hline ASD12 & $6^{*}$ & $30^{*}$ & $0^{*}$ & $0^{*}$ & $0^{*}$ & $0^{*}$ & $0^{*}$ & $0^{*}$ \\
\hline ASD14 & $0^{*}$ & $30^{*}$ & $0^{*}$ & $0^{*}$ & $0^{*}$ & $0^{*}$ & $0^{*}$ & $0^{*}$ \\
\hline ASD22 & $0^{*}$ & $30^{*}$ & $0^{*}$ & $0^{*}$ & $0^{*}$ & $0^{*}$ & $0^{*}$ & $0^{*}$ \\
\hline ASD23 & $0^{*}$ & $30^{*}$ & $0^{*}$ & $0^{*}$ & $0^{*}$ & $0^{*}$ & $0^{*}$ & $0^{*}$ \\
\hline ASD25 & $0^{*}$ & $30^{*}$ & $0^{*}$ & $0^{*}$ & $0^{*}$ & $0^{*}$ & $0^{*}$ & $0^{*}$ \\
\hline ASD29 & $0^{*}$ & $30^{*}$ & $0^{*}$ & $0^{*}$ & $0^{*}$ & $0^{*}$ & $0^{*}$ & $0^{*}$ \\
\hline ASD31 & $0^{*}$ & $30^{*}$ & $0^{*}$ & $0^{*}$ & $0^{*}$ & $0^{*}$ & $0^{*}$ & $0^{*}$ \\
\hline
\end{tabular}

Note: ${ }^{*}$ indicates performance below the mean performance of TD children on standardised measures. 